\title{
Exploring the Tan contact term in Yang-Mills theory
}

\author{
Ouraman Hajizadeh, ${ }^{1}$ Markus Q. Huber $\odot,{ }^{2}$ Axel Maas $\odot,{ }^{1}$ and Jan M. Pawlowski ${ }^{3,4}$ \\ ${ }^{1}$ Institute of Physics, NAWI Graz, University of Graz, Universitätsplatz, 5, A-8010 Graz, Austria \\ ${ }^{2}$ Institut für Theoretische Physik, Justus-Liebig-Universität Giessen, \\ Heinrich-Buff-Ring 16, 35392 Giessen, Germany \\ ${ }^{3}$ Institut für Theoretische Physik, Universität Heidelberg, Philosophenweg 16, \\ 69120 Heidelberg, Germany \\ ${ }^{4}$ ExtreMe Matter Institute EMMI, GSI, Planckstraße 1, 64291 Darmstadt, Germany
}

(Received 8 October 2019; accepted 5 February 2021; published 25 February 2021)

\begin{abstract}
Reliably computing the free energy in a gauge theory like QCD is a challenging and resource-demanding endeavor. We explore the possibility to obtain the associated thermodynamic anomaly from two-point functions based on a conjectured relation. This conjecture is triggered by the relation to the Tan contact in condensed matter systems. For this investigation we use state-of-the-art results for the Yang-Mills gluon two-point function from the lattice and the functional renormalization group, as well as novel DysonSchwinger results at finite temperature computed in the present work. This allows for a first, qualitative, test of this conjecture. The results from all methods reveal the same nontrivial temperature behavior of the subleading large momentum dependence of the gluon propagator relevant for the conjectured relation. The comparison with the expected behavior for SU(2) Yang-Mills theory is encouraging to further pursue this approach.
\end{abstract}

DOI: 10.1103/PhysRevD.103.034023

\section{INTRODUCTION}

Thermodynamic observables including, e.g., density correlations are among the most prominent observables that provide information about the phase structure of heavy-ion collisions. Their computations rely on access to the bulk thermodynamic information of the system. This information is encoded in the free energy, whose determination at finite temperature and density is of prime interest. At large densities functional approaches such as the functional renormalization group (FRG) and Dyson-Schwinger equations (DSEs) circumvent the eminent sign problem that at present prevents lattice simulations in this regime. However, while the part of correlations and thermodynamics that comes from the matter fluctuations does not pose problems in functional approaches, the access to the thermodynamics of gauge fluctuations poses a formidable challenge beyond perturbation theory. At its root it is related to the relevant momentum-scale running of the thermodynamic potentials such as the free energy. Even though by now functional methods have reached high quantitative precision, such a computation still remains a

Published by the American Physical Society under the terms of the Creative Commons Attribution 4.0 International license. Further distribution of this work must maintain attribution to the author(s) and the published article's title, journal citation, and DOI. Funded by SCOAP ${ }^{3}$. demanding calculation in terms of resources. This asks for approaches that reduce the computational effort.

Such an alternative may be provided by a computation in terms of the Tan contact [1,2]; for a discussion in YangMills theory see Ref. [3]. Essentially, it boils down to the idea, reviewed in Sec. II, that the thermodynamic part could be encoded in a simple way in the high-momentum behavior of the two-point correlation functions. These are much simpler to determine reliably. While the extracted part still needs some processing to obtain the free energy, drastic features, e.g., phase transitions, should manifest themselves already directly in the unprocessed data. The aim of the present work is to explore exactly this possibility.

To this end, we use the Landau gauge gluon propagator of SU(2) Yang-Mills theory at finite temperature. This theory undergoes a second-order phase transition at a wellestablished critical temperature. Furthermore, its free energy is known quite well. It is thus an ideal test bed for a new method. We use for this purpose the gluon propagator as obtained using lattice methods, the functional renormalization group, and Dyson-Schwinger equations. The results are shown in Sec. III.

The Tan contact term is related in a straightforward way to the thermodynamic anomaly. This is exploited in Sec. IV, where in a proof-of-principle style the anomaly is determined. There it will also be discussed what further steps are required to make this a quantitatively competitive approach.

In fact, the results show that interesting features of the thermodynamics are already manifest for the unprocessed 
data. These results are encouraging in that this is possible and may be a promising future avenue, as is concluded in Sec. V.

\section{SETUP}

The basic idea of how to transfer the Tan contact term formalism [1,2] from solid state physics and ultracold atoms (e.g., Refs. [4-8]) to particle physics has been outlined in Ref. [3]. It essentially boils down to the fact that the high-momentum behavior of a propagator $D\left(T, p_{0}, \vec{p}\right)$, i.e., at momenta $p \gg \Lambda_{\mathrm{YM}}$, depending on both the temperature $T$ and the momentum $p$, should behave essentially as

$$
D\left(T, p_{0}^{2}, \vec{p}^{2}\right) \stackrel{p^{2} / T^{2} \rightarrow \infty}{\longrightarrow} \frac{Z}{\frac{1}{D_{0}\left(p_{0}^{2}+\vec{p}^{2}\right)}+\Delta \Gamma_{T}\left(p_{0}^{2}, \vec{p}^{2}\right)},
$$

with

$\Delta \Gamma_{T}=\Delta m_{\infty}^{2}(T)+C(T) D_{0}\left(p_{0}^{2}+\vec{p}^{2}\right)+O\left(\frac{1}{p^{4}}\right)$.

In Eq. (1), $D_{0}$ is the vacuum propagator and $Z$ is a total normalization of the propagator.

The coefficient $C(T)$ is the analogue of the Tan contact term in condensed matter systems as introduced in Ref. [3]. By construction, the Tan contact term satisfies $C(T=0)=$ 0 if the fit form (1) describes the propagator perfectly. Note also that the Tan contact in Eq. (1) is not RG invariant: it runs with twice the anomalous dimension of the propagator. An RG-invariant form is easily achieved by multiplication with the wave function renormalization squared. As we concentrate on the comparison between functional approaches and the lattice, this is not important for us.

The temperature-dependent term $\Delta m_{\infty}^{2}(T)$ is a potential contribution to the inverse propagator for asymptotically large momenta. In the present work we consider the gluon propagator, but it should be noted that this term is not to be confused with the chromoelectric and chromomagnetic thermal screening masses. The Slavnov-Taylor identity (STI) (gauge consistency) together with regularity excludes a term $\Delta m_{\infty}^{2}$ in the vacuum; for discussions see Ref. [9-16]. In functional methods, one typically employs a momentum cutoff in the UV. With this numerically convenient choice of regularization, the STI may be satisfied only approximately within truncations, i.e., $\Delta m_{\infty}^{2}(T=0) \approx 0$.

At finite $T$, the STIs cannot be used straightforwardly to exclude such a term. However, lattice results at asymptotic momenta (see Appendix A) show that it is negligible compared to other thermodynamic scales. Consequently, it does not affect the qualitative behavior of the Tan contact term, and we drop it from now on. This also entails that the presence of such terms in the functional results would be a truncation artifact. We have checked that $\Delta m_{\infty}^{2}(T)$ is negligible for the transverse (scaling) propagators which is in line with the observation of gauge consistency of the scaling solution in the vacuum [14,16,17]. In turn, the longitudinal propagator shows a larger $\Delta m_{\infty}^{2}(T) / T$. Still, this contribution is significantly smaller than the Debye mass. In summary, we neglect these terms in the following.

As we are interested in high energies, we set $D_{0}$ to be the one-loop resummed gluon propagator

$$
D_{0}\left(p^{2}, \mu^{2}\right)=\frac{1}{p^{2}\left(1+\omega^{2} \ln \frac{p^{2}}{\mu^{2}}\right)^{\frac{13}{22}}},
$$

which entails $Z=1$. The quantity $\omega$ also involves the coupling $g$. To accommodate for different renormalization prescriptions, we fit $\omega^{2}$ to the zero-temperature propagator for the different methods rather than use some prescribed value. This approach describes the gluon propagator above $2 \mathrm{GeV}$ at zero temperature for all methods at the 1-2\% level. In this regime also the propagators from all methods coincide at this level of precision.

In addition, the thermal gluon propagator splits into a longitudinal chromoelectric one and a transverse chromomagnetic one with respect to the four-velocity of the heat bath. Accordingly, we use Eq. (1) independently for both, thus computing a chromoelectric and chromomagnetic Tan contact, $C_{L}(T)$ and $C_{\perp}(T)$ respectively. To be in the asymptotic regime, we use only data above $|\vec{p}|>2 \mathrm{GeV}$ and the zeroth Matsubara frequency, though at these energies the approximation $D\left(T, p_{0}^{2}, \vec{p}^{2}\right) \approx D\left(T, 0, p_{0}^{2}+\right.$ $\vec{p}^{2}$ ) holds well anyway [18].

For the lattice case, we use the data from Ref. [19] with some additional statistics and two additional lattice discretizations at $T / T_{c}=0.9$ and $T / T_{c}=1.1$ with an $8 \times 40^{3}$ lattice. For the zero-temperature form (2) data from Ref. [20] are used. This entails statistical errors on the fit parameters $Z$ and $\omega$, which were propagated to the fit of $C(T)$. While $\omega=0.82_{-0.03}^{+0.04}$ is essentially $\beta$ independent, $Z$ was interpolated for different $\beta$ values by $Z_{0} 1.50_{-0.03}^{+0.06}(\ln \beta)^{-1.11_{-0.04}^{+0.01}}$, where $Z_{0}$ is the arbitrarily chosen renormalization prescription at zero temperature at fixed $\mu=2 \mathrm{GeV}$. In all cases fits where done along spatial diagonals, which are least affected by discretization effects at high momenta [20].

For the FRG, we use the results from Ref. [15]. The vacuum results yield $Z=2.69 Z_{0}$ and $\omega=0.795$ at $\mu=2 \mathrm{GeV}$. The value for $\omega$ agrees well with the lattice result.

We also extract the Tan contact term from DSE results. Although they are obtained from a much simpler truncation than the FRG results, the high-momentum behavior is determined sufficiently well to extract the relevant information as shown below. Details of the DSE calculations can be found in Appendix B. Their fit parameters are $Z=$ $1.78 Z_{0}$ and $\omega=0.752$, again in good agreement with the 
other methods. The respective gluon propagators are the most modern finite-temperature Yang-Mills ones obtained from Dyson-Schwinger equations and constitute an important step towards a full DSE analysis of QCD without further input at finite temperature and density. Older results were reviewed in Ref. [11]. For a calculation based on a variational approach see Ref. [21].

The temperatures are taken from the respective works as well, i.e., we did not additionally try to fix any scales independently. This yields agreement of the spatialdiagonal lattice data and the DSE and FRG results from $2 \mathrm{GeV}$ up to $12 \mathrm{GeV}$ at the percent level and thus for the whole range of relevant momenta in this work.

\section{RESULTS}

At finite temperature, we find that the fits work well at the few-percent level in all cases. However, results from the lattice for the two temperatures $T / T_{c}=0.9$ and $T / T_{c}=$ 1.1 with 10 times more statistics reveal that Eq. (1) is insufficient if at that level of statistics a subpercent fit is desired. Rather, $C(T)$ needs then to be replaced by some extended form, e.g., $C(T)+p^{2} D(T)$; see also Appendix A. A similar result is obtained in the FRG and DSE cases. However, for the present purpose, and without a major effort for creating more statistics for the lattice, we content ourselves here with fits at the 2-3\% level, which at low statistics is also the statistical accuracy of the lattice results, allowing for agreement within errors. Note that for the continuum results the fit stops working above roughly $T / T_{c} \approx 5$. This is expected, as when $T$ becomes larger, eventually screening effects will propagate to larger momenta which are not included in the fit ansatz (1).

The results are shown in Fig. 1. First of all, it is visible that the general agreement between lattice and functional methods is satisfactory, except for the DSE in the transverse

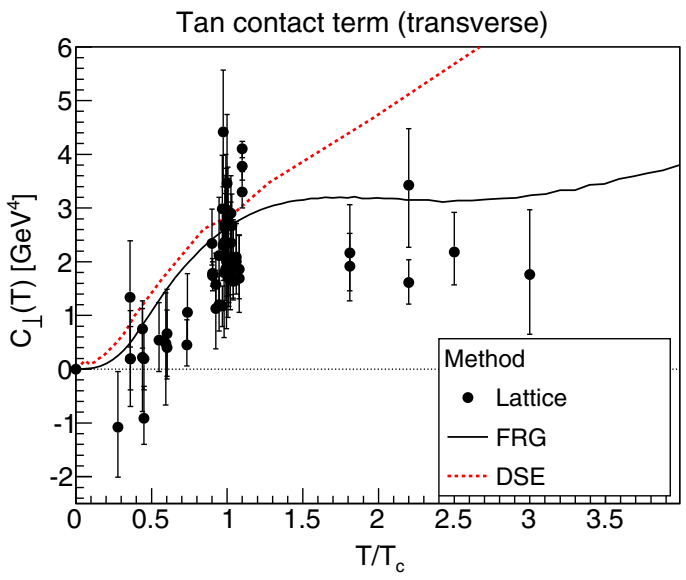

(a) case at high temperatures. Also, at this level of statistics no statistically significant dependency on lattice parameters is visible. Then, there are a number of visible trends which are quite different for the transverse and the longitudinal Tan contact term.

The probably most significant one is the difference between the transverse one and the longitudinal one at high temperatures. The transverse one starts to rise from essentially zero somewhere around $t=T / T_{c} \approx 0.8$ for the lattice data, levels off shortly after $t \gtrsim 1$, and stays constant up to $t \approx 3$. There is no significant change happening at the phase transition. The functional results switch on smoothly, but follow the same trend. However, above $t \gtrsim 2.5$, the functional methods yield again a slow rise of the Tan contact term.

The longitudinal one is quite different. Up to $t \approx 1$, the lattice results are compatible with zero. There is a slight systematic, though not statistically satisfactory trend to nonzero values above $t=1$. However, at large temperatures the Tan contact term rises quicker than quadratically with temperature. Except for the smoothing of the transition, this behavior is also seen in the functional results, this time with no particular impact at $t \gtrsim 3$.

In comparison to the low-momentum behavior [15,19], this provides a consistent picture. There, the transverse propagator also shows no substantial impact of the phase transition, while the longitudinal one seems to do so. At the same time, the impact at high temperatures is also stronger for the longitudinal one.

This leads us to the following picture: the transverse sector carries nontrivial thermodynamic behavior, which is sensitive to the interactions which create a strongly interacting phase above the phase transition for a range of a few $T_{c}$. The bulk thermodynamics is manifested in the longitudinal degrees of freedom, including both the phase transition and the Stefan-Boltzmann trend at high temperatures.

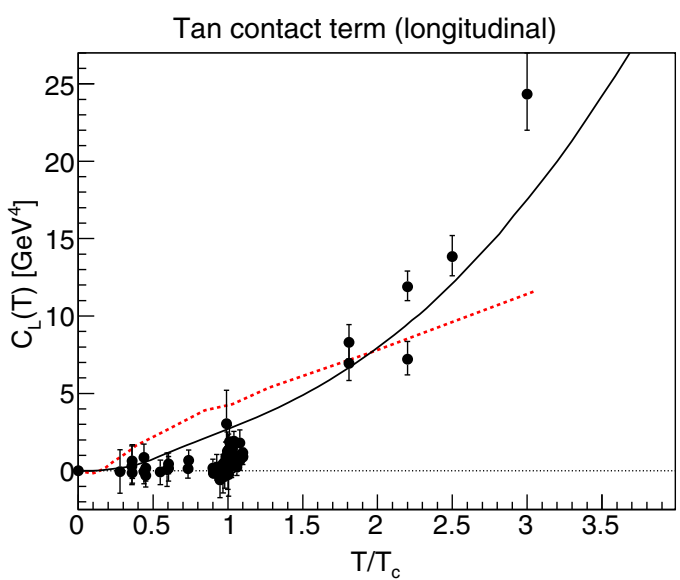

(b)

FIG. 1. Tan contact of Yang-Mills propagators from the lattice, the FRG and DSEs. (a) Transverse $C_{\perp}(T)$ as a function of the temperature. (b) Longitudinal $C_{L}(T)$ as a function of temperature. 


\section{ANOMALY FROM THE TAN CONTACT TERM}

While the Tan contact term in solid state physics and ultracold atoms encodes the thermodynamics, it is in itself not yet equivalent to a thermodynamic potential. However, it is linked to the thermodynamic anomaly $A(T)$ (see e.g., Ref. [22]),

$$
A(T)=\beta(g(T)) C(T)
$$

wherein $\beta(g)$ is the $\beta$ function and $g(T)$ the temperaturedependent running coupling evaluated at the temperature. An analogous derivation in Yang-Mills theory faces several intricacies. First of all this concerns the unphysical nature of gluon fields in comparison to that in solid state and ultracold atomic systems. This leads us to negative norm states in the Fock space as well as the occurrence of ghost fields. Accordingly, a Yang-Mills analogue of the relation (3) will involve $C_{\perp}, C_{L}$ and $C_{\text {ghost }}$ and the respective $\beta$ functions $\beta_{\perp}, \beta_{L}, \beta_{\text {ghost }}$ as well as additional normalization factors. The latter differ in the strongly correlated lowtemperature regime with $T \lesssim T_{c}$. Being short of the full resolution of the different ingredients of the Yang-Mills relation we here discuss the chromomagnetic and chromoelectric parts of this relation. They are given by

$$
A_{\perp / L}(T) \approx-\beta(g(T)) C_{\perp / L}(T),
$$

where we will use the same $\beta$ function for chromomagnetic and chromoelectric parts and take the normalization factors to unity.

Note that the left-hand side of Eq. (4) is related to an observable: the thermodynamic or trace anomaly in YangMills theory, derived from the expectation value of the trace of the energy-momentum tensor (EMT). The expectation value trace and similarly correlation functions of the EMT can be computed within functional methods from closed

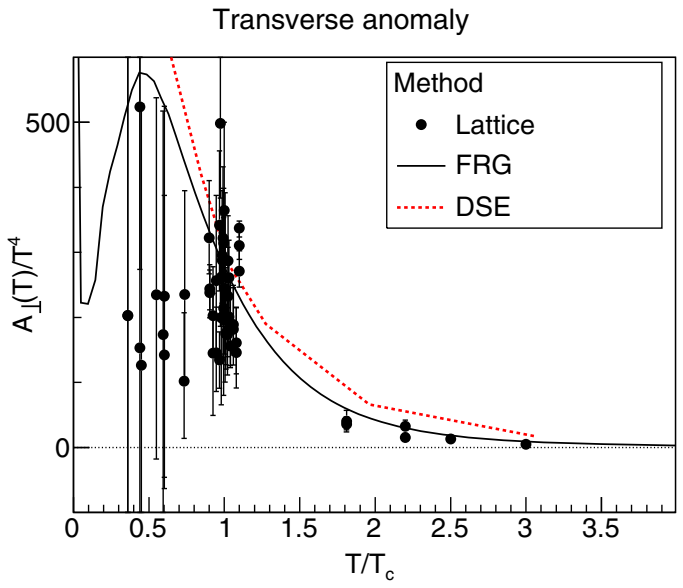

(a) diagrammatic formulas; see Refs. [23,24] for the two-point correlation function of the EMT. These diagrammatic representations are proportional to $\alpha_{s}(T)=g^{2}(T) /(4 \pi)$ [and its scale derivative $\beta(g(T))$ ], where $\alpha_{s}(T)$ is linked to scheme-dependent vertex couplings.

However, the left-hand side of Eq. (4) is scheme independent and hence the scheme dependences on the righthand side need to cancel. This implies that the Tan contact term is scheme dependent. In addition, the miniMOM or Taylor scheme $[25,26]$ employed in the calculation of the gluon propagators yields a multivalued $\beta$ function and its precise determination in lattice calculations requires high statistics. While the former can be remedied by using the temperature-dependent correct branch, the latter precludes us yet from a full determination within each method separately. Also, as will be seen, the Tan contact term needs to be determined at much higher precision in the low-temperature domain.

However, as a proof of principle, we will use here an analytic, temperature-independent coupling motivated by analytic perturbation theory,

$$
\alpha(p)=\pi \frac{\ln \frac{\Lambda_{c}^{2}}{\Lambda_{\mathrm{YM}}^{2}}}{\ln \frac{\Lambda_{c}^{2}+p^{2}}{\Lambda_{\mathrm{YM}}^{2}}}
$$

taking $\Lambda_{c}^{2}=1.21 \mathrm{GeV}^{2}$ for the cutoff momentum and $\Lambda_{\mathrm{YM}}^{2}=0.81 \mathrm{GeV}^{2}$ for the scale. Similar and more elaborate fits have been used within functional methods for vertex couplings as present in the computations of correlations of the EMT. For the theoretical setup, definitions and analytic fits see, e.g., Refs. [14,16,25-40]. Note that the use of Eq. (5) will necessarily upset the overall scale of the result as we do not use matched schemes. The choice of coupling here has little qualitative impact; see Appendix C.

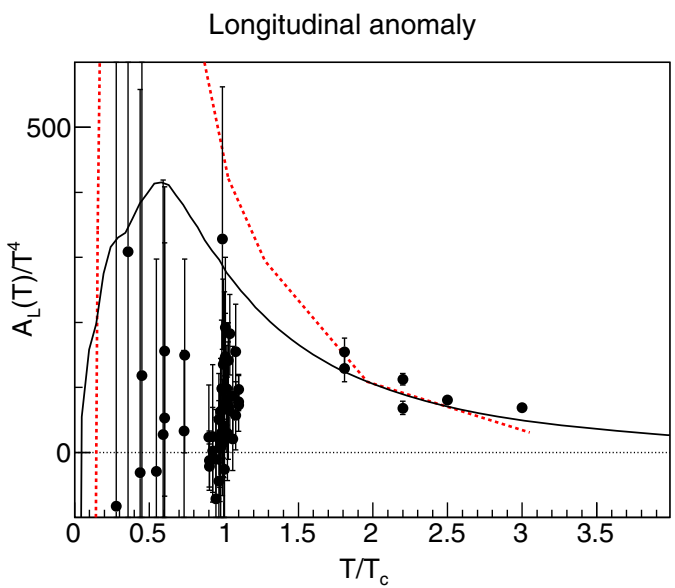

(b)

FIG. 2. The anomaly obtained from the Tan contact term from the lattice, the FRG and DSEs. (a) Transverse anomaly $A_{\perp}(T)$ as a function of the temperature. (b) Longitudinal anomaly $A_{L}(T)$ as a function of temperature. 
The results are shown in Fig. 2. The lattice results, albeit with large errors, are consistent with the temperature dependence of the anomaly, showing a peak around the phase transition, and a slow decrease towards large temperatures. At low temperatures, the Tan contact term is compatible with zero within the errors, and so is necessarily the anomaly. Moreover, we deduce from Fig. 2 that the overall normalization of Eq. (4) is nontrivial as the trace anomaly $A_{\mathrm{YM}}$ in Yang-Mills obeys $A_{\mathrm{YM}} \lesssim 3$ (see e.g., Ref. [41]), while $A_{\perp / L} \lesssim 10^{3}$. Both functional results show a quite similar behavior at high temperatures, but tend to have the peak at far too low temperatures. This is likely partly because this temperature regime is in the deep infrared, where the $\beta$ function is not dominated by its perturbative behavior. Here, a determination of the $\beta$ function in a consistent scheme would likely cure these problems.

Nonetheless, the anomaly shows qualitatively the expected behavior, indicating that the Tan contact term may indeed be a suitable approach to obtain thermodynamic information from propagators.

\section{CONCLUSIONS}

We have extracted for the first time the Tan contact term for Yang-Mills theory from the gluon propagator. We see that known thermodynamic features, the phase transition, the asymptotic Stefan-Boltzmann behavior, and the strongly interacting liquid behavior, imprint themselves qualitatively in the Tan contact term. We also see that the various effects distribute themselves among the transverse and longitudinal degrees of freedom differently. While the strong-interaction regime above the phase transition seems to be encoded in the chromomagnetic sector, the critical and bulk behavior seems to be carried by the chromoelectric sector. This agrees with observations in the infrared [19]. It has also been shown that it is, in principle, possible to use the Tan contact term to determine the anomaly and thus thermodynamic bulk properties.

The obvious steps to be taken from here are to improve statistics and systematics on the lattice and to compare to further results from other sources, e.g., hard-thermal loop calculations or results from dimensionally reduced calculations $[42,43]$. Another issue is contributions from the ghost, which at first sight seems to be inert to temperature $[15,19,44,45]$. For a reconstruction of the thermodynamic potential in full it is required to find the correct normalization, a suitable scheme and sufficient precision to determine the anomaly. Finally, an extension to finite density is of high interest. Here, QCD-like theories without a sign problem, e.g., two-color $\mathrm{QCD}$ or $\mathrm{G}_{2}-\mathrm{QCD}$, could also be interesting testing grounds.

\section{ACKNOWLEDGMENTS}

We are grateful to T. Enss and N. Wink for discussions and a critical reading of the manuscript. We thank A. K. Cyrol for many discussions and a collaboration in an early phase of the project. O. H. was supported by the Austrian Science Fund (FWF) Doctoral Program W1203 "Hadrons in vacuum, nuclei and stars" and by the mobility program of the Science Faculty of the University of Graz. M.H. was supported by the FWF under Contract No. P27380-N27. The work is supported by ExtreMe Matter Institut, the German Federal Ministry of Education and Research (BMBF) Grant No. 05P18VHFCA, and is part of and supported by the DFG Collaborative Research Centre "SFB 1225 (ISOQUANT)" as well as by Deutsche Forschungsgemeinschaft (DFG) under Germany's Excellence Strategy EXC-2181/1-390900948 (the Heidelberg Excellence Cluster STRUCTURES). O.H., M.H., and A.M. are grateful for the warm hospitality at the University of Heidelberg during their visits there.

\section{APPENDIX A: ULTRAVIOLET PROPERTIES AT FINITE TEMPERATURE}

Gauge invariance in terms of the STIs forbids constant terms such as $\Delta m_{\infty}^{2}$ in the vacuum. For more details in the present context see Refs. [9-16]. $\Delta m_{\infty}^{2}=0$ can be implemented in the vacuum in functional methods and holds trivially on the lattice. At finite temperature, gauge invariance, or rather gauge consistency in terms of the STIs, cannot be used straightforwardly to show the existence or disappearance of $\Delta m_{\infty}^{2}$. Moreover, if present, $\Delta m_{\infty}^{2}$ is not necessarily the same for transverse and longitudinal propagators. We also emphasize that the $\Delta m_{\infty}^{2}$ (defined for $\vec{p}^{2} \rightarrow \infty$ ) are not the magnetic or Debye screening masses.

We have investigated the transverse and longitudinal $\Delta m_{\infty}^{2}$ terms on the lattice, which are shown in Fig. 3. The propagators approach the vacuum-resummed one-loop behavior well for the whole temperature range used in this work: both, the transverse and longitudinal $\Delta m_{\infty}^{2}$, are negligible for a fit in the intermediate-momentum regime used for the extraction of the Tan contacts $C_{\perp / L}(T)$. Consequently, they were dropped.

As in the main text, the data shown in Fig. 3 is restricted to the body-diagonal momenta, which are least affected by discretization artifacts [20]. Nonetheless, there are two kinds of deviations. At the largest momenta, a deviation from the one-loop case seems to occur. However, this is a discretization artifact, which is also seen in the vacuum [20]. In addition, there seems to be a multiplicative offset when extracting the logarithmic behavior. This is due to the fact that for the renormalization the vacuum results are used, as in the main text, which have an aspect ratio of 1 . This is not the case at finite temperature, which introduces an additional effect. It decreases with better discretization, as is visible when making the lattice finer at fixed temperature. Because the contact term in the main text is, within errors, not affected by the discretization, this is irrelevant.

What still could be considered, and is compatible with the data, is a running mass which runs at least as fast as 
Transverse gluon dressing function

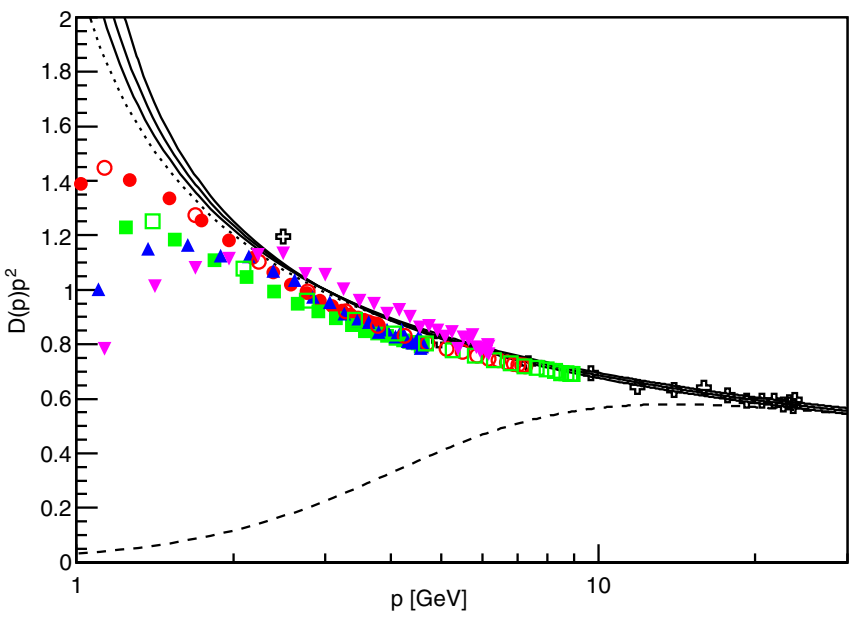

Longitudinal gluon dressing function

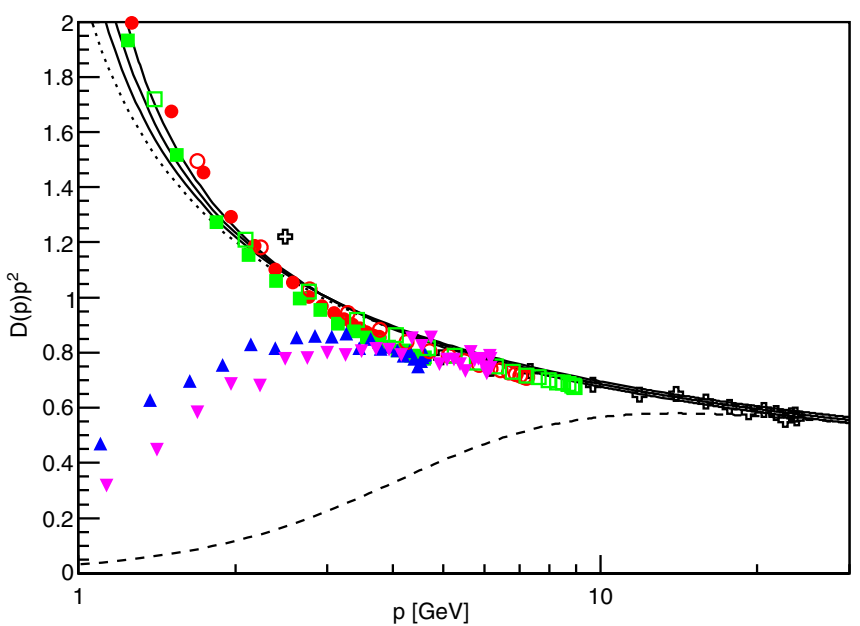

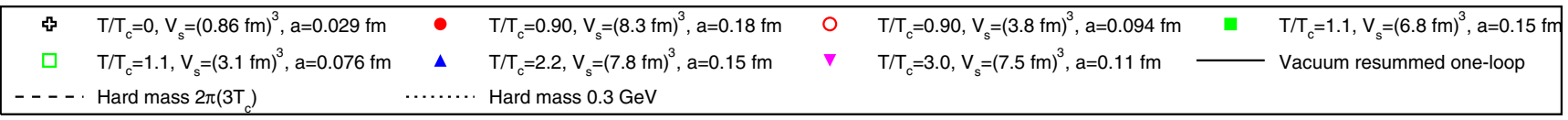

Transverse UV asymptotics

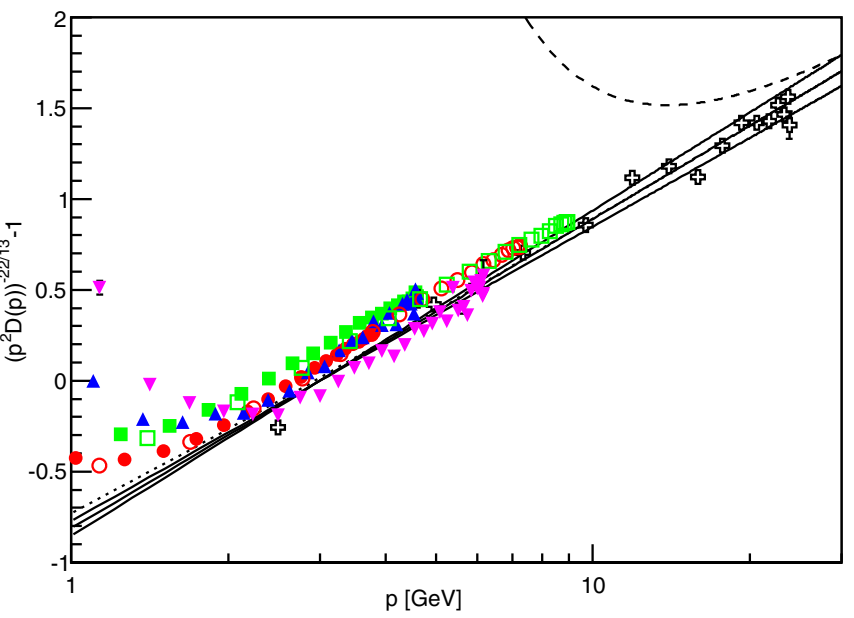

Longitudinal UV asymptotics

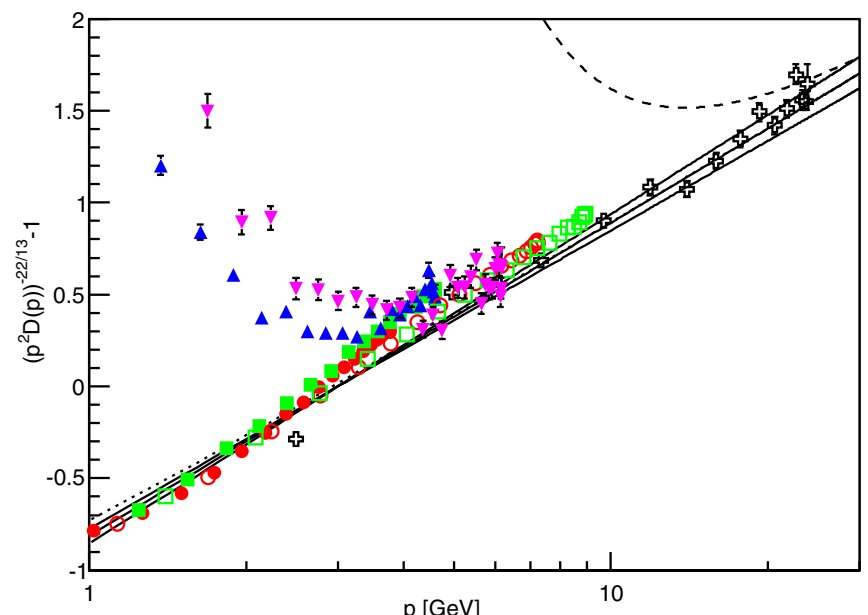

FIG. 3. The high-momentum tail of the transverse (top) and longitudinal (bottom) gluon propagator (left) at various temperatures, compared to the zero-temperature behavior and the resummed one-loop vacuum behavior (2), where the band reflects the uncertainty in $\omega$. The right-hand side extracts the logarithmic running. Results are from Refs. [19,20] with two new additional parameter sets $\left(T / T_{c}=0.9\right.$ and 1.1). The soft modes are shown. Due to the simple relation of hard modes to soft modes at high momentum [18], hard modes behave in the same way. For comparison, deviations from the resummed one-loop behavior with different hard masses are shown in the right column.

the anomalous kinetic part. To investigate this, we considered the possibility

$\Delta \Gamma_{T}=\frac{1}{\left(p_{0}^{2}+\vec{p}^{2}\right) D_{0}\left(p_{0}^{2}+\vec{p}^{2}\right)} \Delta m^{2}(T)+C(T) D_{0}\left(p_{0}^{2}+\vec{p}^{2}\right)$,

i.e., a mass that runs with the same anomalous dimension as the kinetic term. To be able to disentangle the two contributions sufficiently, we use for this purpose only the momentum range $[1.5,4] \mathrm{GeV}$ rather than the range in the main text. Otherwise, the very different momentum running tends to dominate the fit results too much.
Unsurprisingly, with the additional degree of freedom, these fits work even better than the ones in the main text, yielding agreement within statistical errors.

The result is shown in Fig. 4. As is visible, the (by construction) positive-definite mass term is within errors compatible with zero below the phase transition. Above, it steeply rises to a finite value, and remains there. The contact term is reduced, and switches on somewhat later than without the mass term, but shows the same qualitative behavior as in the main text. It is thus clear that the additional degree of freedom in the fit just allows for a split of the temperature dependency of the propagator between both terms, but once the Tan contact term starts to dominate 

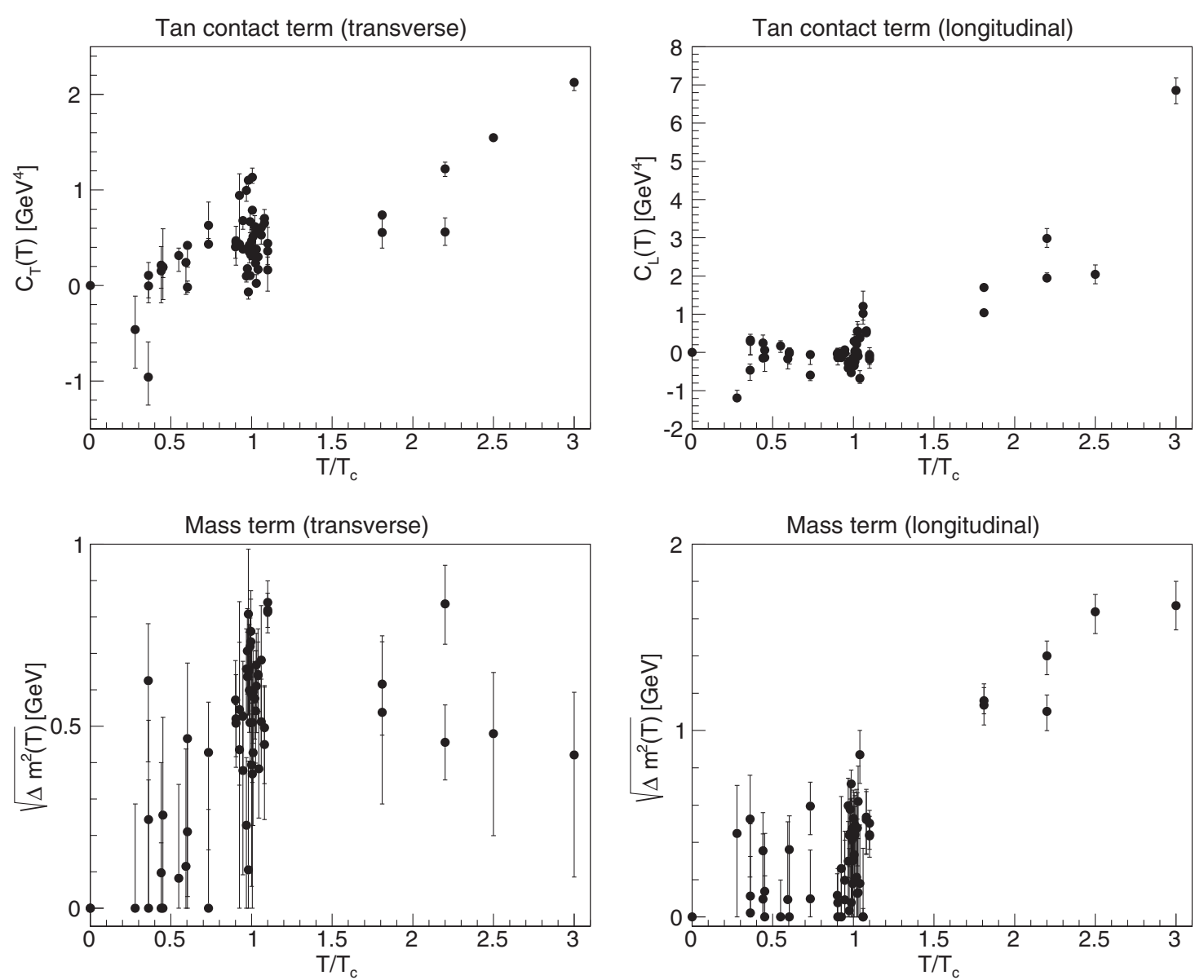

FIG. 4. Results for the extended fit form (A1). Top panels show the Tan contact terms and bottom panels the effective masses. Left panels show the transverse case and right panels the longitudinal case.

at high temperature, it is clearly seen. As there is thus no qualitative influence of more degrees of freedom in the fit, we conclude that the qualitative behavior in the main text is genuine. A true quantitative description would certainly require much better statistics to resolve the momentum dependence, and possibly further subleading terms.

Performing the same for the results from functional methods leads to similar results. Note that in functional approaches truncation artifacts may mix with such terms; for further discussions see Refs. [9-16].

\section{APPENDIX B: PROPAGATORS FROM DYSON-SCHWINGER EQUATIONS}

The truncation used for the calculation of the propagators from their DSEs is described in the following. The equations that were solved are the ones for the ghost and gluon propagators truncated to one-loop without tadpoles. The only remaining higher $n$-point functions are the ghost-gluon and three-gluon vertices. The former is taken as bare, which is within the context of the present work sufficient since we are only interested in the highmomentum behavior. The deviation from a bare vertex is known to be a bump around $1 \mathrm{GeV}$ which falls off quickly $[15,45,46]$.

The three-gluon vertex plays a crucial role for the gluon propagator. It is not only quantitatively relevant, but the existence of a solution for the gluon propagator also depends strongly on its properties. Here, the following model adapted from Ref. [47] was used for dressing the tree-level tensor:

$$
C^{A A A}\left(p_{0}, q_{0}, \vec{p}, \vec{q}\right)=\frac{G\left(\bar{p}^{2}\right)}{Z_{T}\left(\bar{p}^{2}\right)} \frac{\bar{p}^{2}}{\bar{p}^{2}+\Lambda_{s}^{2}}\left(-G\left(\bar{p}^{2}\right)^{3} \frac{\Lambda_{3 \mathrm{~g}}^{2}}{\Lambda_{3 \mathrm{~g}}^{2}+p^{2}} \frac{\Lambda_{3 \mathrm{~g}}^{2}}{\Lambda_{3 \mathrm{~g}}^{2}+q^{2}} \frac{\Lambda_{3 \mathrm{~g}}^{2}}{\Lambda_{3 \mathrm{~g}}^{2}+(p+q)^{2}}+\frac{G\left(\bar{p}^{2}\right)}{Z_{T}\left(\bar{p}^{2}\right)} \frac{\bar{p}^{2}}{\bar{p}^{2}+\Lambda_{s}^{2}}\right)
$$




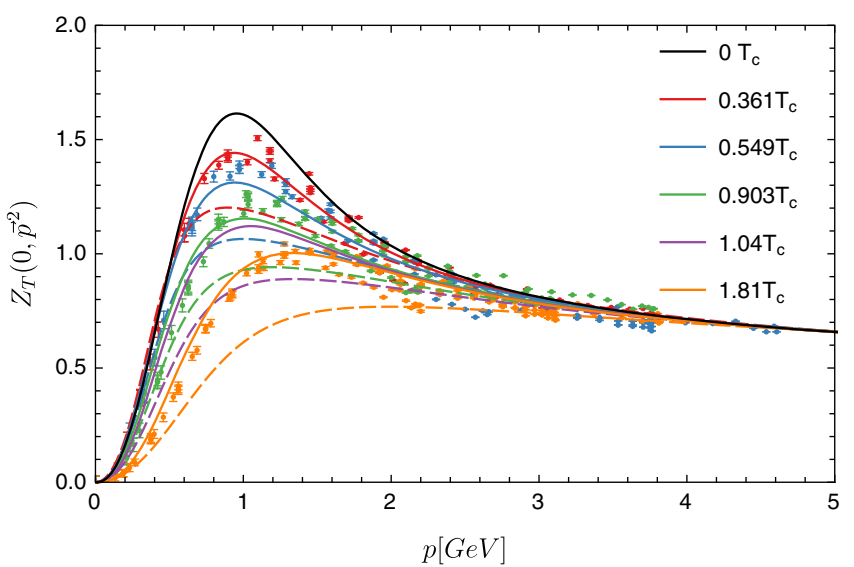

(a)

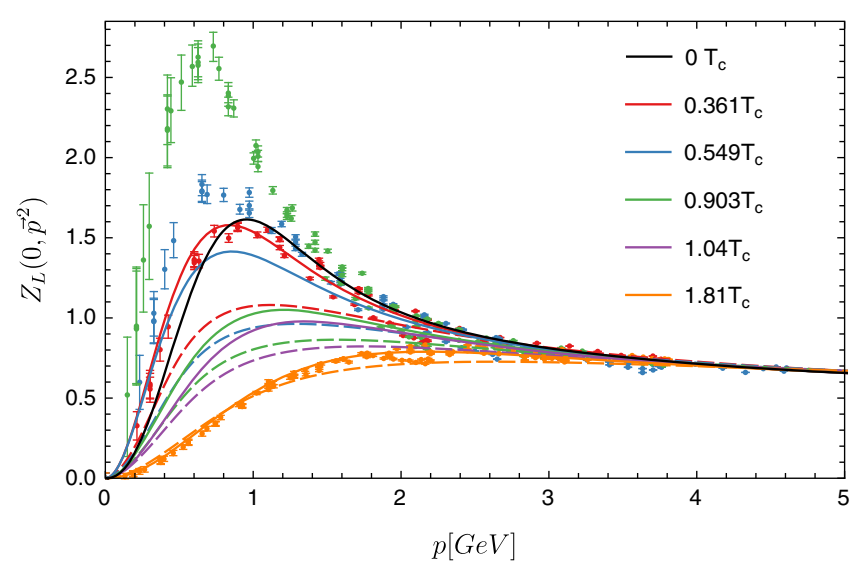

(b)

FIG. 5. Gluon propagator dressings for selected temperatures from the FRG (continuous), DSEs (dashed) and lattice simulations $[18,19]$. For reference the vacuum result from the FRG is shown in black. (a) Chromo-magnetic gluon propagator dressings for selected temperatures. (b) Chromo-electric gluon propagator dressings for selected temperatures.

The momentum $\bar{p}^{2}$ is $\left(p^{2}+q^{2}+(p+q)^{2}\right) / 2$ and $p$ and $q$ are four-momenta. $G$ and $Z_{T}$ are the ghost and the transverse gluon dressing functions, respectively. The first term in the parentheses determines the IR behavior of the vertex, and the second the UV behavior. The term in front of the parentheses accounts for missing perturbative higherloop contributions relevant for the resummed one-loop behavior $[40,48,49]$. The model contains two scales which are fixed as $\Lambda_{s}=\Lambda_{3 \mathrm{~g}}=0.741 \mathrm{GeV}$.

The integral kernels for the Dyson-Schwinger equations can be found, e.g., in Ref. [50]. Here they were derived with DoFun [51-53], and the equations were solved with CrasyDSE [54]. Quadratic divergences in the gluon propagator DSE were renormalized via second renormalization conditions chosen as the value of the propagators at zero momentum $[40,55,56]$. For the employed truncation this leaves some ambiguity in how to select these conditions, but at the scales of relevance here it is expected that such effects are subleading.

The overall scale was set for the lowest calculated temperature by matching the UV tail to FRG results. The relative scales for the other temperatures were set by matching the perturbative couplings.

The resulting dressing functions for the gluon propagators are shown in Fig. 5 for selected temperatures. Clearly, the present truncation cannot capture the IR behavior but reproduces the momentum and temperature dependencies qualitatively. Both, in the FRG and the DSE computation of the propagators the computation of the nontrivial $A_{0}$ background has not been taken into account. This background $A_{0} \neq 0$ is the equation of motion and is directly linked to the vanishing of the Polyakov loop in the confining phase [57-61]; for perturbative computations within the background see Ref. [62].

In Ref. [15] it has been argued that this should lead to deviations of the chromoelectric propagators in functional approaches from the chromoelectric lattice propagators (as they are computed on different a background) for temperatures with

$$
0.5 T_{c} \lesssim T \lesssim 1.3 T_{c} .
$$

Indeed this expectation is confirmed by the data; see Fig. 5(b).

\section{APPENDIX C: INFLUENCE OF THE RUNNING COUPLING ON THE ANOMALY}

The anomaly (3) depends on the choice of coupling, which, in turn, depends on a choice of scheme. Of course, at sufficiently high temperature where the expression $\beta(g(T))$ is dominated by the first two perturbative terms all results will coincide. That is expected to happen above a temperature of roughly $2 \mathrm{GeV} \approx 6.7 T_{c}$. In addition, for temperatures below $0.5 T_{c}$ the Tan contact term is essentially zero, and thus the behavior of the running coupling becomes irrelevant as well.

To illustrate the situation, consider a running coupling consistent with our FRG results, which is fitted with very high accuracy by a modified fit form from Ref. [63] given by

$$
\alpha(p)=\frac{\left(0.314 p^{4}+0.877 p^{2}+1\right)\left(0.935 p^{2}\left(\frac{1}{\log \left(0.818 p^{2}\right)}-\frac{1}{0.818 p^{2}-1}\right)+0.0229\right)}{0.302 p^{6}+0.0983 p^{4}+0.179 p^{2}+0.00745} .
$$




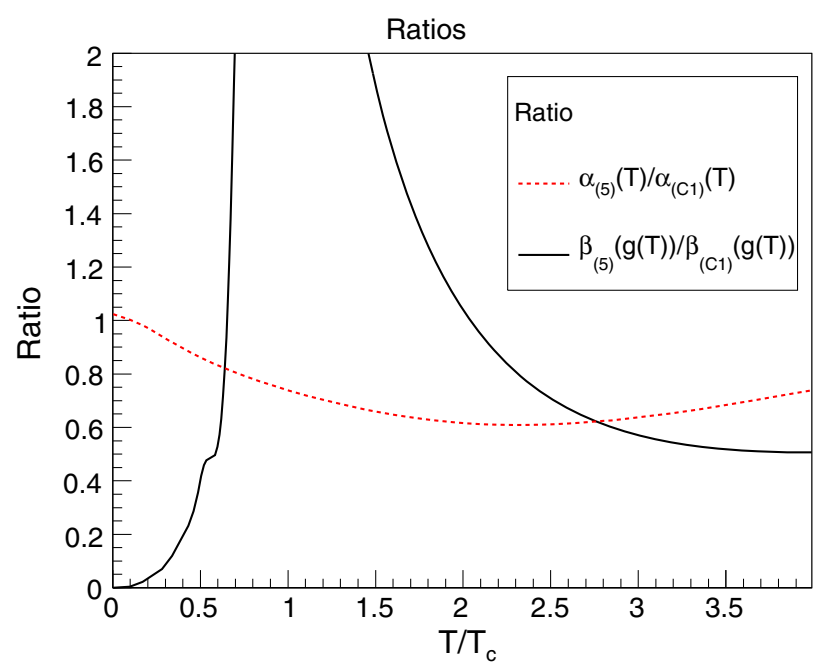

FIG. 6. Ratios of the running couplings and the corresponding $\beta(g(T))$ functions of Eqs. (5) and (C1).
Units on the fit coefficients have been suppressed. The ratio of this coupling to the one used in the main text [Eq. (5)] and of the relevant function $\beta(g(T))$ in Eq. (4) is shown in Fig. 6. The difference at temperatures above $1.5 T_{c}$ is just a few tens of percent, with little dependence on the temperature. The relative sizes at large temperatures are dominated by the different runnings and therefore the ratio approaches one slowly. The situation is more drastic below this temperature, as here the running coupling $(\mathrm{C} 1)$ has a maximum around $p \approx 270 \mathrm{MeV}$ and is thus double valued. Here we always chose the positive branch. More severe is that the $\beta$ function has a zero crossing. This implies that the anomaly would vanish at this temperature. The existence of this maximum is, however, a subtle question, and appears to be scheme dependent. This makes it clear that the relative scheme dependence of the Tan contact term in relation to the running coupling needs to be resolved to get a quantitative result for the anomaly.
[1] S. Tan, Energetics of a strongly correlated Fermi gas, Ann. Phys. (Amsterdam) 323, 2952 (2008).

[2] S. Tan, Generalized virial theorem and pressure relation for a strongly correlated fermi gas, Ann. Phys. (Amsterdam) 323, 2987 (2008).

[3] L. Fister and J. M. Pawlowski, Yang-mills correlation functions at finite temperature, arXiv:1112.5440.

[4] J. T. Stewart, T. E. Gaebler, and D. S. Jin, Verification of Universal Relations in a Strongly Interacting Fermi Gas, Phys. Rev. Lett. 104, 235301 (2010).

[5] B. Mukherjee, P. B. Patel, Z. Yan, R. J. Fletcher, J. Struck, and M.W. Zwierlein, Spectral Response and Contact of the Unitary Fermi Gas, Phys. Rev. Lett. 122, 203402 (2019).

[6] T. Enss, R. Haussmann, and W. Zwerger, Viscosity and scale invariance in the unitary fermi gas, Ann. Phys. (Amsterdam) 326, 770 (2011).

[7] I. Boettcher, S. Diehl, J. M. Pawlowski, and C. Wetterich, Tan contact and universal high momentum behavior of the fermion propagator in the BCS-BEC crossover, Phys. Rev. A 87, 023606 (2013).

[8] E. Braaten, Universal relations for fermions with large scattering length, in The BCS-BEC Crossover and the Unitary Fermi Gas, Lecture Notes in Physics Vol. 836 (Springer, New York, 2012), p. 193.

[9] U. Ellwanger, Flow Equations and BRS Invariance for Yang-Mills Theories, Phys. Lett. B 335, 364 (1994).

[10] A. Maas, J. Wambach, and R. Alkofer, The high-temperature phase of Landau-gauge Yang-Mills theory, Eur. Phys. J. C 42, 93 (2005).

[11] A. Maas, Describing gauge bosons at zero and finite temperature, Phys. Rep. 524, 203 (2013).
[12] D. Binosi, D. Ibanez, and J. Papavassiliou, The all-order equation of the effective gluon mass, Phys. Rev. D 86, 085033 (2012).

[13] M. Q. Huber and L. von Smekal, Spurious divergences in Dyson-Schwinger equations, J. High Energy Phys. 06 (2014) 015.

[14] A. K. Cyrol, L. Fister, M. Mitter, J. M. Pawlowski, and N. Strodthoff, Landau gauge Yang-Mills correlation functions, Phys. Rev. D 94, 054005 (2016).

[15] A. K. Cyrol, M. Mitter, J. M. Pawlowski, and N. Strodthoff, Nonperturbative finite-temperature Yang-Mills theory, Phys. Rev. D 97, 054015 (2018).

[16] M. Q. Huber, Correlation functions of Landau gauge YangMills theory, Phys. Rev. D 101, 114009 (2020).

[17] A. K. Cyrol, M. Mitter, J. M. Pawlowski, and N. Strodthoff, Non-perturbative quark, gluon and meson correlators of unquenched QCD, Phys. Rev. D 97, 054006 (2017).

[18] C. S. Fischer, A. Maas, and J. A. Müller, Chiral and deconfinement transition from correlation functions: SU(2) vs. SU(3), Eur. Phys. J. C 68, 165 (2010).

[19] A. Maas, J. M. Pawlowski, L. von Smekal, and D. Spielmann, The gluon propagator close to criticality, Phys. Rev. D 85, 034037 (2012).

[20] A. Maas, Some more details of minimal-Landau-gauge Yang-Mills propagators, Phys. Rev. D 91, 034502 (2015).

[21] M. Quandt and H. Reinhardt, A covariant variational approach to Yang-Mills Theory at finite temperatures, Phys. Rev. D 92, 025051 (2015).

[22] T. Enss, Bulk Viscosity and Contact Correlations in Attractive Fermi Gases, Phys. Rev. Lett. 123, 205301 (2019). 
[23] M. Haas, L. Fister, and J. M. Pawlowski, Gluon spectral functions and transport coefficients in Yang-Mills theory, Phys. Rev. D 90, 091501 (2014),

[24] N. Christiansen, M. Haas, J. M. Pawlowski, and N. Strodthoff, Transport Coefficients in Yang-Mills Theory and QCD, Phys. Rev. Lett. 115, 112002 (2015),

[25] L. von Smekal, K. Maltman, and A. Sternbeck, The strong coupling and its running to four loops in a minimal MOM scheme, Phys. Lett. B 681, 336 (2009).

[26] P. Boucaud, F. De Soto, J. P. Leroy, A. Le Yaouanc, J. Micheli, O. Pène, and J. Rodríguez-Quintero, Ghost-gluon running coupling, power corrections and the determination of $\Lambda_{\overline{\mathrm{MS}}}$, Phys. Rev. D 79, 014508 (2009),

[27] T. Kugo and I. Ojima, Local covariant operator formalism of nonabelian gauge theories and quark confinement problem, Prog. Theor. Phys. Suppl. 66, 1 (1979).

[28] J. M. Cornwall, Dynamical mass generation in continuum QCD, Phys. Rev. D 26, 1453 (1982).

[29] B. Alles, D. S. Henty, H. Panagopoulos, C. Parrinello, C. Pittori, and D. G. Richards, $\alpha_{s}$ from the nonperturbatively renormalised lattice three gluon vertex, Nucl. Phys. B502, 325 (1997),

[30] L. von Smekal, R. Alkofer, and A. Hauck, The Infrared Behavior of Gluon and Ghost Propagators in Landau Gauge QCD, Phys. Rev. Lett. 79, 3591 (1997).

[31] R. Alkofer and L. von Smekal, The infrared behavior of QCD Green's functions: Confinement, dynamical symmetry breaking, and hadrons as relativistic bound states, Phys. Rep. 353, 281 (2001).

[32] A. C. Aguilar, A. A. Natale, and P. S. Rodrigues da Silva, Relating a Gluon Mass Scale to an Infrared Fixed Point in Pure Gauge QCD, Phys. Rev. Lett. 90, 152001 (2003).

[33] R. Alkofer, C. S. Fischer, and F. J. Llanes-Estrada, Vertex functions and infrared fixed point in Landau gauge SU(N) Yang-Mills theory, Phys. Lett. B 611, 279 (2005).

[34] C.S. Fischer, Infrared properties of QCD from DysonSchwinger equations, J. Phys. G 32, R253 (2006).

[35] C. S. Fischer, A. Maas, and J. M. Pawlowski, On the infrared behavior of Landau gauge Yang-Mills theory, Ann. Phys. (Amsterdam) 324, 2408 (2009).

[36] A. C. Aguilar, D. Binosi, J. Papavassiliou, and J. RodriguezQuintero, Non-perturbative comparison of QCD effective charges, Phys. Rev. D 80, 085018 (2009).

[37] A. C. Aguilar, D. Binosi, and J. Papavassiliou, QCD effective charges from lattice data, J. High Energy Phys. 07 (2010) 002.

[38] G. Eichmann, R. Williams, R. Alkofer, and M. Vujinovic, Three-gluon vertex in Landau gauge, Phys. Rev. D 89, 105014 (2014).

[39] A. Deur, S. J. Brodsky, and G. F. de Teramond, The QCD running coupling, Prog. Part. Nucl. Phys. 90, 1 (2016).

[40] M. Q. Huber, Nonperturbative properties of Yang-Mills theories, Phys. Rep. 879, 1 (2020).

[41] S. Borsanyi, G. Endrodi, Z. Fodor, S. D. Katz, and K. K. Szabo, Precision SU(3) lattice thermodynamics for a large temperature range, J. High Energy Phys. 07 (2012) 056.

[42] J.-P. Blaizot and E. Iancu, The quark-gluon plasma: Collective dynamics and hard thermal loops, Phys. Rep. 359, 355 (2002).
[43] A. Hietanen, K. Kajantie, M. Laine, K. Rummukainen, and Y. Schröder, Three-dimensional physics and the pressure of hot QCD, Phys. Rev. D 79, 045018 (2009).

[44] M. Q. Huber and L. von Smekal, On two- and three-point functions of Landau gauge Yang-Mills theory, Proc. Sci. LATTICE2013 (2014) 364 [arXiv:1311.0702].

[45] L. Fister and A. Maas, Exploratory study of the temperature dependence of magnetic vertices in SU(2) Landau gauge Yang-Mills theory, Phys. Rev. D 90, 056008 (2014).

[46] M. Q. Huber, An exploratory study of Yang-Mills threepoint functions at non-zero temperature, EPJ Web Conf. 137, 07009 (2017).

[47] M. Q. Huber, On non-primitively divergent vertices of Yang-Mills theory, Eur. Phys. J. C 77, 733 (2017).

[48] L. von Smekal, A. Hauck, and R. Alkofer, A solution to coupled Dyson-Schwinger equations for gluons and ghosts in Landau gauge, Ann. Phys. (N.Y.) 267, 1 (1998).

[49] M. Q. Huber and L. von Smekal, On the influence of threepoint functions on the propagators of Landau gauge YangMills theory, J. High Energy Phys. 04 (2013) 149.

[50] A. Maas, The high-temperature phase of Yang-Mills theory in Landau gauge, PhD thesis, Darmstadt University of Technology, 2004.

[51] R. Alkofer, M. Q. Huber, and K. Schwenzer, Algorithmic derivation of Dyson-Schwinger equations, Comput. Phys. Commun. 180, 965 (2009).

[52] M. Q. Huber and J. Braun, Algorithmic derivation of functional renormalization group equations and DysonSchwinger equations, Comput. Phys. Commun. 183, 1290 (2012).

[53] M. Q. Huber, A. K. Cyrol, and J. M. Pawlowski, DoFun 3.0: Functional equations in mathematica, Comput. Phys. Commun. 248, 107058 (2020).

[54] M. Q. Huber and M. Mitter, CrasyDSE: A framework for solving Dyson-Schwinger equations, Comput. Phys. Commun. 183, 2441 (2012).

[55] J. C. Collins, Renormalization: An Introduction to Renormalization, the Renormalization Group, and the Operator Product Expansion (Cambrdige University Press, Cambridge, England, 1984).

[56] J. Meyers and E. S. Swanson, The gluon propagator with two-loop Schwinger-Dyson equations, Phys. Rev. D 90, 045037 (2014).

[57] J. Braun, H. Gies, and J. M. Pawlowski, Quark confinement from color confinement, Phys. Lett. B 684, 262 (2010).

[58] F. Marhauser and J. M. Pawlowski, Confinement in Polyakov gauge, arXiv:0812.1144.

[59] J. Braun, A. Eichhorn, H. Gies, and J. M. Pawlowski, On the nature of the phase transition in $\mathrm{SU}(\mathrm{N}), \mathrm{Sp}(2)$ and $\mathrm{E}(7)$ Yang-Mills theory, Eur. Phys. J. C 70, 689 (2010).

[60] L. Fister and J. M. Pawlowski, Confinement from correlation functions, Phys. Rev. D 88, 045010 (2013).

[61] T. K. Herbst, J. Luecker, and J. M. Pawlowski, Confinement order parameters and fluctuations, arXiv:1510.03830.

[62] U. Reinosa, J. Serreau, M. Tissier, and A. Tresmontant, Yang-Mills correlators across the deconfinement phase transition, Phys. Rev. D 95, 045014 (2017).

[63] R. Alkofer, C. S. Fischer, and L. von Smekal, Infrared exponents and the running coupling of Landau gauge QCD and their relation to confinement, Eur. Phys. J. A 17, 773 (2003). 